\title{
REPRESENTING A CLOSED OPERATOR AS A QUOTIENT OF CONTINUOUS OPERATORS
}

\author{
WILLIAM E. KAUFMAN
}

\begin{abstract}
The closed operators in a Hilbert space $H$ are characterized as quotients $A B^{-1}$ of continuous operators on $H$ such that the vector sum $A^{*}(H)+B^{*}(H)$ is closed. This leads to the function $\Gamma(A)=A(1-$ $\left.A^{*} A\right)^{-1 / 2}$, which is shown to map the strictly contractive operators on $H$ reversibly onto the closed densely-defined operators, so as to preserve the selfadjoint and nonnegative conditions.
\end{abstract}

Introduction. Suppose that $(H,\langle\cdot, \cdot\rangle)$ is a complete complex inner product space (e.g. a Hilbert space) with corresponding norm $\|\cdot\|$ such that for each $x$ in $H,\|x\|=\langle x, x\rangle^{1 / 2}$, and let $(H \times H,\langle\cdot, \cdot\rangle)$ denote the usual product space with corresponding norm $\|\cdot\|^{\sim}$. By a closed operator in $H$ is meant a linear function from a linear subspace of $H$ into $H$ which is closed in $H \times H$. Let $\mathscr{B}(H)$ denote the algebra of all continuous linear functions from $H$ into $H$ ("including" the complex numbers) and for each $A$ in $\mathscr{B}(H)$, let $A^{*}$ denote the adjoint of $A$, let $A^{-1}$ denote the inverse of the restriction of $A$ to the closure of $A^{*}(H)$, and-in case $A$ is nonnegative-let $A^{-1 / 2}$ denote $\left(A^{1 / 2}\right)^{-1}$. Note that for each $A$ in $\mathscr{B}(H), A^{-1} A$ is the orthogonal projection from $H$ onto the closure of $A^{*}(H)$.

THEOREM 1. If $C$ is a subset of $H \times H$ then the following two statements are equivalent:

(1) $C$ is a closed operator in $H$, and

(2) $C$ is the quotient $A B^{-1}$ of a member $(A, B)$ of $\mathscr{B}(H) \times \mathscr{B}(H)$ such that the vector sum $A^{*}(H)+B^{*}(H)$ is closed in $H$.

Proof. Suppose (1) is true. Let $S$ denote the domain of $C$ and for all $(x, y)$ in $S \times S$, let

$$
\langle x, y\rangle^{\prime}=\langle(x, C x),(y, C y)\rangle^{\sim} .
$$

Since $C$ is linear and closed, $\left(S,\langle\cdot, \cdot\rangle^{\prime}\right)$ is a complete inner product space; moreover, for each $x$ in $S$,

$$
\|x\|^{2} \leqslant\langle x, x\rangle^{\prime} \text { and }\|C x\|^{2} \leqslant\langle x, x\rangle^{\prime} .
$$

It follows from a result of Mac Nerney [3, Theorem 3, p. 666] that there is a nonnegative member $B$ of $\mathscr{B}(H)$ such that $B(H)=S$ and for each $(x, y)$ in

Received by the editors November 22, 1977 and, in revised form, February 21, 1978.

AMS (MOS) subject classifications (1970). Primary 47A45, 47A65.

Key words and phrases. Closed operator, Hilbert space, complete inner product space, strictly contractive operator, quotients of operators, Cayley transform.

(1) American Mathematical Society 1978 
$S \times S,\langle x, y\rangle^{\prime}=\left\langle B^{-1} x, B^{-1} y\right\rangle$. (An argument appears in the proof in [4, p. 110].) Let $A=C B$ : if $x$ is in $H$ then

$$
\|A x\|^{2} \leqslant\langle B x, B x\rangle^{\prime} \leqslant\|x\|^{2} .
$$

Thus $A$ is in $\Re(H)$. Now let $P$ denote the orthogonal projection $B^{-1} B$. Then $A B^{-1}=C, A P=A$, and for all $(x, y)$ in $H \times H$,

$$
\left\langle x,\left[B^{2}+A^{*} A\right] y\right\rangle=\langle(B x, C B x),(B y, C B y)\rangle^{\sim}=\langle B x, B y\rangle^{\prime}=\langle x, P y\rangle .
$$

Hence $B^{2}+A^{*} A=P$, and since $A^{*}(H)+B(H)=\left[B^{2}+A^{*} A\right]^{1 / 2}(H)[1$, Theorem 2.2, p. 260], it follows that $A^{*}(H)+B(H)$ is closed. Therefore (2) is true.

Now suppose (2), and let $P=B^{-1} B$ as before. We first show that $C$ is also the quotient $(A P) B^{-1}$, and that $(A P)^{*}(H)+B^{*}(H)$ is also closed-so that it suffices to consider only the case in which $A P=A$. Clearly $C=(A P) B^{-1}$, since $B B^{-1}$ is simply the identity function on $B(H)$. Suppose $z$ is a member of the closure of $(A P)^{*}(H)+B^{*}(H)$. The fact that $(A P)^{*}=P A^{*}$ implies that $z$ is in the closure of $B^{*}(H)$ and therefore in the closed set $A^{*}(H)+$ $B^{*}(H)$. Thus there is a member $(x, y)$ of $A^{*}(H) \times B^{*}(H)$ for which $x+y=$ $z$. Since each of $y$ and $z$ is in the closure of $B^{*}(H)$, so is $x$. Hence $P x=x$, so that $x$ is in $P A^{*}(H)$ and therefore $z$ is in $(A P)^{*}(H)+B^{*}(H)$; the latter set must therefore be identical with its closure.

Suppose now that $A P=A$. Note that in this case, the function whose value at each $x$ in $H$ is $(B x, A x)$-which maps the closure of $B^{*}(H)$ reversibly onto $C$-also maps $H$ onto $C$. Let $D$ denote $\left[A^{*} A+B^{*} B\right]^{1 / 2}$ and consider the linear function $J$ defined on the closed set $A^{*}(H)+B^{*}(H)$ by

$$
J(z)=\left(B D^{-1} z, A D^{-1} z\right), \text { for all } z \text { in } D(H) .
$$

Since $A^{*}(H)+B^{*}(H)$ includes the closure of $B^{*}(H), J$ maps the former onto $C$. The following calculation for each $z$ in $D(H)$ shows that $J$ is an isometry from $A^{*}(H)+B^{*}(H)$ onto $C$ :

$$
\|J z\|^{2}=\left\|B D^{-1} z\right\|^{2}+\left\|A D^{-1} z\right\|^{2}=\left\langle D^{-1} z,\left[B^{*} B+A^{*} A\right] D^{-1} z\right\rangle=\|z\|^{2} \text {. }
$$

Thus $C$ is closed-i.e., (1) holds true. This completes the proof.

The first half of the proof provides a construction of such a pair $(A, B)$ as in (2) with certain additional properties, as indicated in the following:

COROLlaRY. If $C$ is a closed operator in $H$ then $C$ is the quotient $A B^{-1}$ of a pair $(A, B)$ of $\mathscr{B}(H) \times \mathscr{B}(H)$ such that $B$ is nonnegative, $A B^{-1} B=A, C$ has domain $B(H)$ and range $A(H)$, and $A^{*} A+B^{2}$ is the orthogonal projection $B^{-1} B$ onto the closure of $B(H)$-which closure is the set $A^{*}(H)+B(H)$.

REMARK. If $C=A B^{-1}$ as in the preceding corollary then the orthogonal projections from $H \times H$ onto the subsets $C$ and $C^{*}$ thereof, respectively, are easily computed as

$$
\left[\begin{array}{ll}
B^{2} & B A^{*} \\
A B & A A^{*}
\end{array}\right] \text { and }\left[\begin{array}{ll}
1-A A^{*} & A B \\
B A^{*} & 1-B^{2}
\end{array}\right]
$$


The formula for the projection onto $C$ is a slight extension of that given by von Neumann in [7, Appendix II, pp. 72-73] for the case in which $C$ has domain dense in $H$-so that $C^{*}$ is a function and the selfadjoint operator $1+C^{*} C$ is available. These connections are elaborated upon in Theorem 2.

Notation. Let $\mathfrak{V}(H)$ denote the subset of $\mathscr{B}(H)$ to which $A$ belongs only in case $A$ is a contraction (i.e., $\|A x\|<\|x\|$ for all nonzero $x$ in $H$ ), and let $\mathcal{C}(H)$ denote the set of all closed operators in $H$ with domain dense in $H$.

THEOREM 2. The equations

$$
\Gamma(A)=A\left(1-A^{*} A\right)^{-1 / 2}, \text { for all } A \text { in } \mathcal{V}(H),
$$

define a reversible function $\Gamma$ from $\mathfrak{V}(H)$ onto $\mathcal{C}(H)$ with inverse function defined by

$$
\Gamma^{-1}(C)=C\left[\left(1+C^{*} C\right)^{-1}\right]^{1 / 2}, \text { for all } C \text { in } \mathcal{C}(H) .
$$

Moreover, $\Gamma$ maps certain subsets of $\mathfrak{V}(H)$ into $\mathcal{C}(H)$ as follows:

(i) the set of all members of $\mathfrak{V}(H)$ with norm less than 1 is mapped onto $\mathscr{B}(H)$,

(ii) the set of all Hermitian (symmetric) members of $\mathcal{V}(H)$ is mapped onto the set of all selfadjoint operators in $H$, and

(iii) the set of all nonnegative members of $\mathfrak{V}(H)$ is mapped onto the set of all nonnegative selfadjoint operators in $H$.

Proof. Suppose $A$ is in $\checkmark(H)$ and let $B$ denote the positive member $\left(1-A^{*} A\right)^{1 / 2}$ of $\mathcal{V}(H)$. Then $\Gamma(A)=A B^{-1}$ and $A^{*} A+B^{2}=1$; therefore by Theorem $1, \Gamma(A)$ is a closed operator in $H$. Since the domain of $\Gamma(A)$ is $B(H)$-which is dense in $H$ (B is positive), $\Gamma(A)$ belongs to $\mathcal{C}(H)$.

Now suppose $C$ is in $\mathcal{C}(H)$. By the corollary to Theorem 1 , there is an $(A, B)$ in $\mathscr{B}(H) \times \mathscr{B}(H)$ such that $C=A B^{-1}, B$ is positive $(B$ has range the domain of $C$ ), and $A^{*} A+B^{2}=1$-i.e., $B=\left(1-A^{*} A\right)^{1 / 2}$. For each nonzero $x$ in $H,\|x\|^{2}-\|A x\|^{2}=\|B x\|^{2}>0$; thus $A$ is in $\widetilde{V}(H)$ and $\Gamma(A)=$ $C$. Now $C$ is the set of all $(B z, A z)$ for $z$ in $H$, so $C^{*}$ is all $(x, y)$ in $H \times H$ such that $\langle x, A z\rangle=\langle y, B z\rangle$ for all $z$ in $H$-equivalently, such that $\left\langle A^{*} x, z\right\rangle$ $=\langle B y, z\rangle$ for all $z$ in $H$. Thus $C^{*}=B^{-1} A^{*}$. Von Neumann has shown [6, p. 301] that $1+C^{*} C$ is reversible with inverse in $\mathscr{B}(H)$ (see also [5, p. 307]), and for each $x$ in $H$ we have that

$$
\begin{aligned}
x & =B^{2} x+B^{-1}\left(1-B^{2}\right) B^{-1} B^{2} x \\
& =\left(1+B^{-1} A^{*} A B^{-1}\right) B^{2} x=\left(1+C^{*} C\right) B^{2} x .
\end{aligned}
$$

Hence $\left(1+C^{*} C\right)^{-1}=B^{2}$, and therefore $A=C B=C\left[\left(1+C^{*} C\right)^{-1}\right]^{1 / 2}$. This establishes that $\Gamma$ is reversible from $\mathscr{V}(H)$ onto $\mathcal{C}(H)$, with inverse as indicated.

To see that (i) is true, observe that for each $A$ in $\mathscr{V}(H)$, there is a positive number $m$ such that $1-A^{*} A \gg m$ only in case $\left(1-A^{*} A\right)^{1 / 2}$ is invertible [5, p. 266], and that this latter holds true provided that $\Gamma(A)$ has domain all of $H$. 
By the closed graph theorem, this is equivalent to $\Gamma(A)$ belonging to $\mathscr{B}(H)$.

The remainder of the proof involves the orthogonal projections described in the preceding remark. First, suppose that $A$ is in $\checkmark(H)$; let $B=(1-$ $\left.A^{*} A\right)^{1 / 2}$ and consider the associated projections from $H \times H$ onto $\Gamma(A)$ and $\Gamma(A)^{*}$. Clearly $\Gamma(A)=\Gamma(A)^{*}$ only in case $B^{2}=1-A A^{*}$ and $A B=B A^{*}$. Since $A^{*} A+B^{2}=1$, this is equivalent to: $A$ is normal (so that $A$ commutes with $B^{2}$ and thus with $B$ ) and $A B=A^{*} B$. Since $B(H)$ is dense in $H$, the latter condition alone is in fact equivalent to $A=A^{*}$. Hence (ii) is true.

Finally, suppose $A$ is an Hermitian member of $\mathcal{V}(H)$, and let $B$ denote $\left(1-A^{2}\right)^{1 / 2}$. Then $A$ commutes with $B^{1 / 2}$, so for each $x$ in $H$,

$$
\left\langle B^{1 / 2} x, A B^{1 / 2} x\right\rangle=\langle B x, A x\rangle=\langle B x, C B x\rangle .
$$

Since $B^{1 / 2}(H)$ is dense in $H, A$ is nonnegative only in case $\langle z, A z\rangle \geqslant 0$ for all $z$ in $B^{1 / 2}(H)$. On the other hand, $C$ is nonnegative only in case $\langle y, C y\rangle \geqslant 0$ for all $y$ in $B(H)$. Thus the preceding equalities imply the equivalence of these two conditions. Therefore (iii) is true.

FURTHER REMARKS. Theorem 2 may be regarded as a refinement of a result of J. J. Koliha, who proves in [2, pp. 778-779] that a member of $\mathscr{B}(H)$ has norm less than one only in case it is $C\left(1+C^{*} C\right)^{-1 / 2}$ for some $C$ in $\Re(H)$. Identification of the function $\Gamma$ now affords us a "natural" topology for $\mathcal{C}(H)$ with respect to which $\mathscr{B}(H)$ is an open set dense therein. Additionally, one may derive therefrom that the vector sum of the domain and range of any selfadjoint operator in $H$ is all of $H$ (via the corollary to Theorem 1).

In conclusion, there is some sharpening of the Cayley transform [5, p. 325]. Let $C=\Gamma(A)$ and $B=\left(1-A^{*} A\right)^{1 / 2}$ as usual. It is easily verified that $C$ is Hermitian only in case $A^{*} B=B A$. In any case, $(C-i)(C+i)^{-1}=(A-$ $i B)(A+i B)^{-1}$-thus the usual representation of the Cayley transform as a quotient of possibly discontinuous operators may be replaced by one such as in Theorem 1. Moreover, $C$ is Hermitian only in case both $A+i B$ and $A-i B$ are linear isometries from $H$ into itself, inasmuch as

$$
\|(A \pm i B) x\|^{2}=\|x\|^{2} \pm i\left\langle x,\left(B A-A^{*} B\right) x\right\rangle,
$$

for all $x$ in $H$.

\section{REFERENCES}

1. P. A. Fillmore and J. P. Williams, On operator ranges, Advances in Math. 7 (1971), 254-271.

2. J. J. Koliha, Convergent and stable operators and their generalizations, J. Math. Anal. Appl. 43 (1973), 778-794.

3. J. S. Mac Nerney, Investigation concerning positive definite continued fractions, Duke Math. J. 26 (1959), 663-678.

4. $109-112$.

, Continuous embeddings of Hilbert spaces, Rend. Circ. Mat. Palmero (2) 19 (1970),

5. F. Riesz and B. Sz.-Nagy, Functional analysis, Ungar, New York, 1955 (transl. of 2nd French ed., 1952).

6. J. von Neumann, Über adjungierte Funktionaloperatoren, Ann. of Math. (2) 33 (1932), 294-310.

7. J. von Neumann, Functional operators, vol. II, Ann. of Math. Studies, no. 22, Princeton Univ. Press, Princeton, N. J., 1950.

Department of Mathematics, University of Houston, Houston, Texas 77004 